\title{
Isopolytungstate Nanocomposite Thermoresponsive Hydrogel for Wastewater Purification
}

\author{
Lili $\mathrm{Li}^{1}$, Cong Wang ${ }^{2 *}$, Yanli Sui ${ }^{*}$, Hui $\mathrm{Li}^{2}$, Yibo Feng ${ }^{2}$ \\ ${ }^{1}$ The state key laboratory for advanced metals and materials, University of Science and Technology Beijing, \\ Beijing, Haidian, China \\ ${ }^{2}$ Institute of Microstructure and Property of Advanced Materials, Beijing University of Technology, \\ Beijing, Chaoyang, China \\ Email: yls@ustb.edu.cn; smartswang@163.com
}

\begin{abstract}
A novel sunlight sensitive composite as a wastewater purification material is presented. The substitute material is a thermoresponsive hydrogel, and the photocatalyst are uniformly dispersed tungsten trioxide nanoparticles. These nanocomposites confining poly(ethylene oxide)poly(propylene oxide)-poly(ethylene oxide) tri-block copolymer (EPE) molecules have packaged visible light driven isopolytungstate nano-buildings within the pores of copolymer fiber nets. The networks are provided (formed) by N-Isopropyl acrylamide (Nipam) and Acrylamide (AM). In wastewater solution, the Nipam-co-AM hydrogel is utilized to be a switchable and efficient filter for larger sized residue when subjected to temperature variation. By adding isopolytungstate nanoparticles as photocatalyst monomer, the small sized toxic organic materials can be effectively degraded. Such a hydrogel with distinct thermal and sunlight responsive properties can be produced as a functional material for wastewater dye degradation and large sized residue treatment of great significance in environmental friendly applications.
\end{abstract}

Keywords: Nanocomposite; Nipam; isopolytungstate; wastewater treatment.

\section{Introduction}

In the past few years, photocatalytic materials have attracted a lot of attention in water pollution treatment, sterilization and water splitting[1-5]. Nowadays, the photocatalytic materials play an important role in the fields of solar energy utilization and environmental purification[6-12]. With the development of photocatalyst technology, there emerged many potential applied strategies for visible light utilization, such as $\mathrm{TiO}_{2}$ nitrogen doping, which can introduce oxygen vacancies, thus tune the material band gap and exhibit stronger visible light absorption[2], and noble metals (Au、 Pt、Ag and so on) doping, where the noble metals usually act as electron acceptors to increase the separation of electrons and holes so as to the improve the photocatalytic performance[13-15]. In addition, the semiconductors are recombined to form a heterojunction or z-scheme structure to enhance photocatalytic performance[16]. Besides, it can be summarized that many functional materials can be used as photocatalysts, including titanium oxide, zinc oxide, tin oxide, zirconium dioxide, cadmium sulfide, and other oxide sulfide semiconductors[12-15, 18]. Eliza et al. demostrated the efficiency of photocatalytic degradation of methyl blue with zinc oxide is significantly improved via the combination of graphene oxide and zinc oxide nanorods[17]. However, photocatalyst is still not widely applied in practical wastewater treatment due to the loss of efficiency and nanoparticles during the recycling process[19]. The smaller sized nanoparticles show higher efficiency in photocatalytic performance. The large specific surface area of the small sized photocatalysts is favourable to the catalytic efficiency enhancement [20].

$\mathrm{WO}_{3}$ is one of the most studied visible light driven photocatalytic materials[11]. Since $\mathrm{WO}_{3}$ has a narrow bandgap $(\mathrm{Eg}=2.6-2.7 \mathrm{eV})$, it is currently a very promising visible light driven photocatalyst[21]. In recent years, photocatalytic materials have been widely used in wastewater treatment, but there is still a long way to meet actual needs. Moreover, In the process of sewage treatment, the problem of dispersion and recovery of small sized materials hinder the current practical applications of tungsten 
oxide. Therefore, we develop a composite route to anchor the particles, so that the small sized photocatalysts can be cycled many times. The raw material we used here is a widely used thermoresponvise polymer named Nipam. Since the cross-linked poly (N-isopropylacrylamide) (PNipam) has a lower critical solution temperature (Tc) at $32-33^{\circ} \mathrm{C}[22]$, The PNipam with other molecules aggregate to form clusters when the temperature is above critical temperature (Tc), which can separate the polymers from water and the mesoporous polymer can filter out large-scale pollutants in water.

In this work, tungsten oxide nanocomposite is used as a photocatalystic part for wastewater treatment. It not only contains the isopolytungstate, but also a PAM/PNipam polymer network. Besides, the triblock-copolymer (EPE) molecules provide small sized isopolytungstate that are uniformly dispersed in water before in-situ polymerization. In wastewater treatment, the PAM/PNipam hydrogel is utilized as a switchable and efficient filter for larger sized residue when subjected to temperature variation. Furthermore, the small sized toxic organic materials can be degraded by adding isopolytungstate as photocatalyst monomer. This is one of the most promising applications in environmental friendly wastewater treatment.

\section{$2 \quad$ Experimental}

\subsection{Materials and Methods}

N,N,N`,N`-Tetramethylethylene diamine (TEMED), Ammonium tungstate, N-Isopropyl acrylamide (Nipam), N,N-Methylene-bisacylamide (Bis), Acrylamide (AM), Ammonium persulfate (Initiator), and poly (ethylene oxide)-poly (propylene oxide)-poly (ethylene oxide) tri-block copolymer (EPE) Ammonium (para) tungstate hydrate, citrate and methyl orange were purchased from Sigma-Aldrich. Scanning Electronic Microscopy S-3000N was from Hitachi. UV-VIS spectrophotometer was Lambda 20 from Perkin Elmer. Memment oven was from Che Scientific Co (Hong Kong) Ltd. Topward DualTracking DC Power supply 6303D. LABCNCO D-090011 freezing drier was from South East Chemicals and Instruments Ltd. We used organic and inorganic composite water-solubility material Nipam+isopolytungstate. All experiments were carried out using deionized, distilled water.

\section{$2.2 \quad$ Synthesis of Photocatalysts}

The photocatalyst is mainly produced from ammonium tungstate surrounded by citric acid (isopolytungstate). An aqueous solution of 8v/v\% EPE (total average molecular weight: 2000; molecular poly (ethylene oxide) (PEO) weight: 1600, sigma) forms a triblock copolymer that can act as a carrier for the tungstate, forming clusters that contain isopolytungstate. Then, $10 \mathrm{v} / \mathrm{v} \%$ thermosensitive Nipam molecules are dissolved into system. $1 \%$ (w/v) precursor solution was prepared by mixing $20 \mathrm{~mL}$ monomer solution, $20 \mu \mathrm{L}$ TEMED, $60 \mu \mathrm{L}$ initiator solution and $0.18 \mathrm{~g}$ EPE molecules, and undergone ultrasonication for 1min, the mixture was kept in the dark for 10 mins. Acrylamide (AM) and N, NMethylene-bisacrylamide (Bis) were dissolved in water to form the monomer solution. The final concentrations of AM in monomer solution prepared were $5 \%(\mathrm{w} / \mathrm{v}), 10 \%(\mathrm{w} / \mathrm{v}), 15 \%(\mathrm{w} / \mathrm{v})$ and $20 \%$ $(\mathrm{w} / \mathrm{v})$ respectively. $0.5 \mathrm{~g} / \mathrm{mL}$ initiator solution was prepared by dissolving $1.0 \mathrm{~g}$ ammonium persulfate in $2 \mathrm{~mL}$ DI water. $2 \mu \mathrm{L}$ TEMED, $6 \mu \mathrm{L}$ initiator solution and $2 \mathrm{~mL}$ monomer solution were mixed together. Then, the mixture was kept in the dark for few minutes where the solution solidified into hydrogel. The hydrogel was cleaned by DI water twice and placed in the refrigerator for 30 mins.

Here, we proposed a new type of thermally responsive hydrogel by adding EPE-isopolytungstate to common PAM/PNipam hydrogel, and this hydrogel was successfully applied in the simulation of wastewater treatment. The gelation conditions were optimized by adjusting the temperature on the molecular size of EPE-isopolytungstate, the pore size and porosity of the PAM/PNipam hydrogel. When the temperature is higher than $30{ }^{\circ} \mathrm{C}$, hydrogel gels.

\subsection{Thermal and near UV Responsive Hydrogel Preparation}

$0.9 \%(\mathrm{w} / \mathrm{v})$ precursor solution was prepared by mixing $20 \mathrm{~mL}$ monomer solution, $20 \mu \mathrm{L}$ TEMED, $60 \mu \mathrm{L}$ initiator solution and $0.18 \mathrm{~g}$ EPE molecules, and the mixture was treated by ultrasonication for $1 \mathrm{~min}$. 
After that it was kept in the dark for 10 mins for hydrogel formation. $0.9 \%$ (w/v) hydrogel precursor solution was pipetted into plastic cuvette. After completed gelation, the plastic cuvette was placed in water bath at a constant temperature and equilibrated for 5 mins. Then, the plastic cuvette was placed in the UV-VIS spectrophotometer and subjected to optical measurement between $300 \mathrm{~nm}$ and $1100 \mathrm{~nm}$. We obtained a series of UV-VIS spectra for hydrogel at different temperatures $\left(20^{\circ} \mathrm{C}, 23^{\circ} \mathrm{C}, 26^{\circ} \mathrm{C}, 30^{\circ} \mathrm{C}\right.$, $\left.40^{\circ} \mathrm{C}\right)$.

\subsection{Photocatalytic Degradation of Methyl Orange}

MO degradation was chosen as a probe reaction to evaluate the photocatalytic activities of this photocatalyst. The concentration of methyl orange is $20 \mathrm{mg} / \mathrm{L}$. Taking the photon utilization under simulated sunlight into account, $50 \mathrm{mg}$ methyl orange solution was used for experiment. The appropriate volume of catalyst hydrogel was added to the methyl orange solution, and the mixture was stirred in the dark and protected from light for $2 \mathrm{~h}$ for uniform mixing.

\section{$3 \quad$ Results and Discussions}

\subsection{Scheme and Mechanism of Thermally-Responsive Photocatalytic Materials}

At room temperature $25{ }^{\circ} \mathrm{C}$, EPE molecules embedding isopolytungstate cluster (the small box in the picture) in the PAM/PNipam fiber are uniformly dispersed in water. At initial stage, the temperature is lower than the critical temperature of $30^{\circ} \mathrm{C}$, hydrogel is transparent to naked eyes shown in Figure 1a. The clusters are uniformly dispersed in the thermal responsive (PAM/PNipam) hydrogel. Due to the photoresponsive property of tungstate in solution under suitable conditions, when it is exposed under $\mathrm{AM}\left(5 \mathrm{~W} / \mathrm{cm}^{2}\right)$, it turns to be blue color immediately, as the Figure $1 \mathrm{~b}$ shows. On the other hand, phase transition of PAM/PNipam occurs when the critical temperature is reached due to its thermal responsive property. Therefore, when the temperature reaches above the critical temperature, PAM/PNipam including EPE molecules aggregated to form clusters (pale blue dots in Figure 1c). The PAM/PNipam with EPE clusters scatter visible light and hydrogel appears as opaque. The phase separation process is reversible after the temperature drops below the critical value.

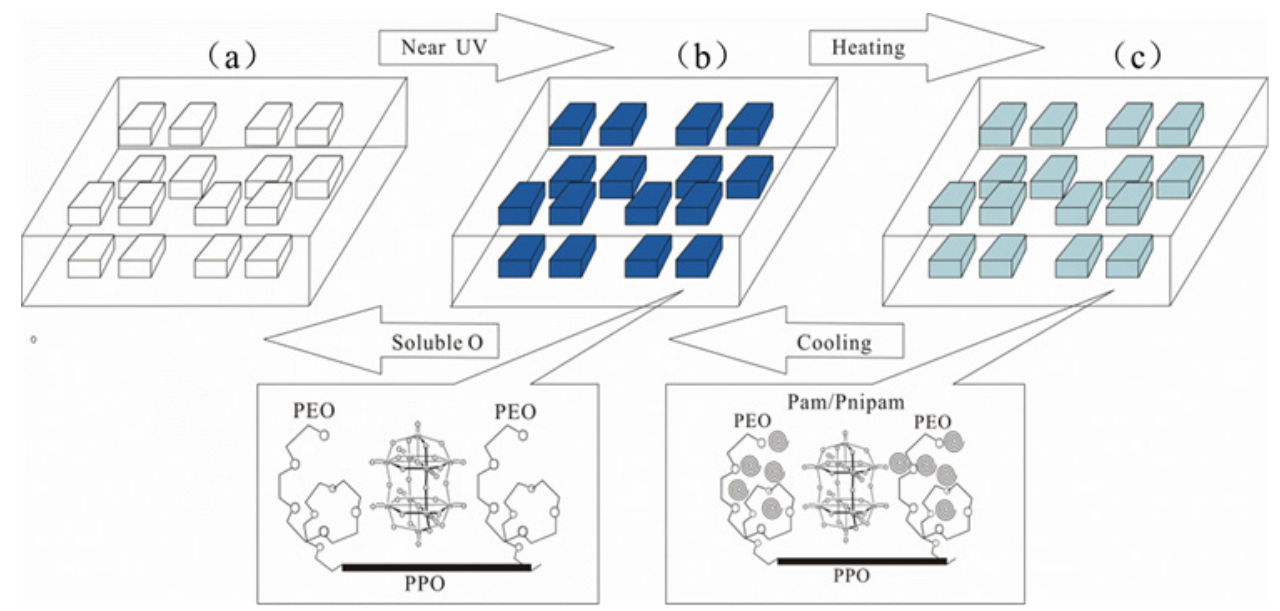

Figure 1. Schematic diagram of the hydrogel thermal response mechanism.

\subsection{Molecule Characterization of EPE Cluster Coordinated with AM and Nipam}

EPE is taken as a carrier to form a small molecular cluster of EPE-isopolytungstate molecules. The size of EPE-isopolytungstate molecules is much smaller than the individual pore size of PAM/PNipam hydrogel, whereas the performance of EPE-isopolytungstate molecules will not be affected. In order to 
figure out the relationship between EPE clusters and PAM/PNipam, the samples were subjected to FTIR test.
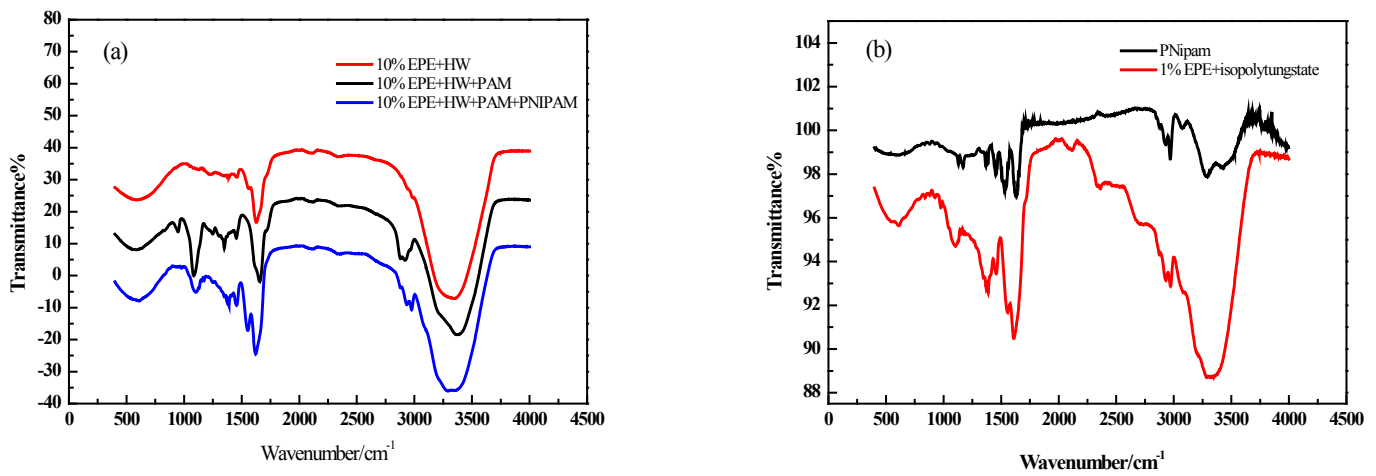

Figure 2. The FTIR distribution of EPE molecule clusters (10\% wt EPE water solution) at different buffers.

According to the obtained FTIR spectra, it is concluded that the structure of the PAM/PNipam hydrogel consists of a sequence of hydrophilic amide group $\left(-\mathrm{CONH}_{2}\right)$ and hydrophobic isopropyl ($\left.\mathrm{CH}\left(\mathrm{CH}_{3}\right)_{2}\right)$. The spectra show that Nipam and AM are cross-linked and no reaction occurs. The intense and broad band appearing at $3300-3030 \mathrm{~cm}^{-1}$ is the $\mathrm{N}-\mathrm{H}$ stretching vibration of ammonium $\mathrm{NH}_{4}^{+}$. Corresponding to the $-\mathrm{OH}$ stretching vibration, an intense and broad band appeared in the region 3200$3600 \mathrm{~cm}^{-1}$. The peaks at $1690 \mathrm{~cm}^{-1}$ represent the $-\mathrm{CONH}_{2}$ amide. The $\mathrm{N}-\mathrm{H}$ is shown up in $3500 \mathrm{~cm}^{-1}$ and $3400 \mathrm{~cm}^{-1}$ in the Figure 2, which may be attributed to the ammonium salt bond. $-\mathrm{CH}_{3}$ asymmetric bending vibrations at $1455 \mathrm{~cm}^{-1}$ and $1175-1165 \mathrm{~cm}^{-1}$, in $-\mathrm{CH}\left(\mathrm{CH}_{3}\right)_{2}$ and $-\mathrm{x}-\mathrm{CH}_{2^{-}}$respectively. The appearance of $560-540 \mathrm{~cm}^{-1}$ may be caused by the stretching vibration of $\mathrm{C}-\mathrm{H}$ in the methyl group on EPE. The $-\mathrm{CH}_{3}$ asymmetric bending are not appeared in EPE+isopolytungstate+PAM curve due to without Nipam molecule in hydrogel. The peaks at $1170 \mathrm{~cm}^{-1}$ and $1113 \mathrm{~cm}^{-1}$ relate to the C-C stretching vibrations of $-\mathrm{CH}\left(\mathrm{CH}_{3}\right)_{2}$ and-CRH- $\mathrm{CH}_{2^{-}}$. In Figure 2a, both black and blue ones have these characteristic peaks, which indicate that Nipam has crosslinked with. Due to that, the molecules constructed together so stablelly that it is possible to be applied in structural material and real industry.

\subsection{Morphology and Porosity Regulation of PAM+PNipam Hydrogel}

After designing the amount of EPE and isopolytungstate, the next step is to select the appropriate hydrogel. Hydrogel is widely used in surface chemistry, separation science and tissue engineering. PAM/PNipam hydrogel is composed of cross-linked polymer fiber grids which can hold up a large amount of water. Compared with other hydrogel extracted from natural products, such as alginate and agarose[23-24], PAM/PNipam hydrogel is stable, reproducible and cheap. More importantly, the polymerization process of AM monomer solution to form the hydrogel will not be affected by the EPE, making PAM/PNipam hydrogel a suitable carrier for EPE molecules[25].

The pore size and porosity of hydrogel have a strong effect on the behavior of EPE molecules. In this work, we regulated porosity by varying the concentration of PAM/PNipam monomer in precursor solution and the degree of cross-linking. We prepared four kinds of precursor solution with $5 \%(\mathrm{w} / \mathrm{v})$, $10 \%(\mathrm{w} / \mathrm{v}), 15 \%(\mathrm{w} / \mathrm{v}), 20 \%(\mathrm{w} / \mathrm{v})$ AM monomer respectively. The weight ratio of (crosslinking agent) was fixed at 19:1, freeze-dried after polymerization. The results show that the higher the Nipam monomer concentration, the lower the porosity of PAM hydrogel. The reduced porosity increases the probability of EPE clusters being trapped in the polymer fibers during the polymerization process and affects the degree of phase transition of the hydrogel. It is found that only hydrogel prepared from $5 \%$ $(\mathrm{w} / \mathrm{v})$ and $10 \%(\mathrm{w} / \mathrm{v})$ AM monomer solution is suitable for our experiment. The PAM hydrogel with a 19:1 weight ratio prepared from the precursor solution is transparent, while the other weight ratios of the PAM hydrogel appeared to be translucent. Therefore, the degree of cross-linking of the polymer fiber affects the light transmittance of the hydrogel. The opaque hydrogel will affect the photocatalytic 
process. The ratio of Nipam, Am and Bis is listed in table 1. As is shown, $10 \% \mathrm{NIPAM}+2.5 \% \mathrm{AM}$ is optimum.
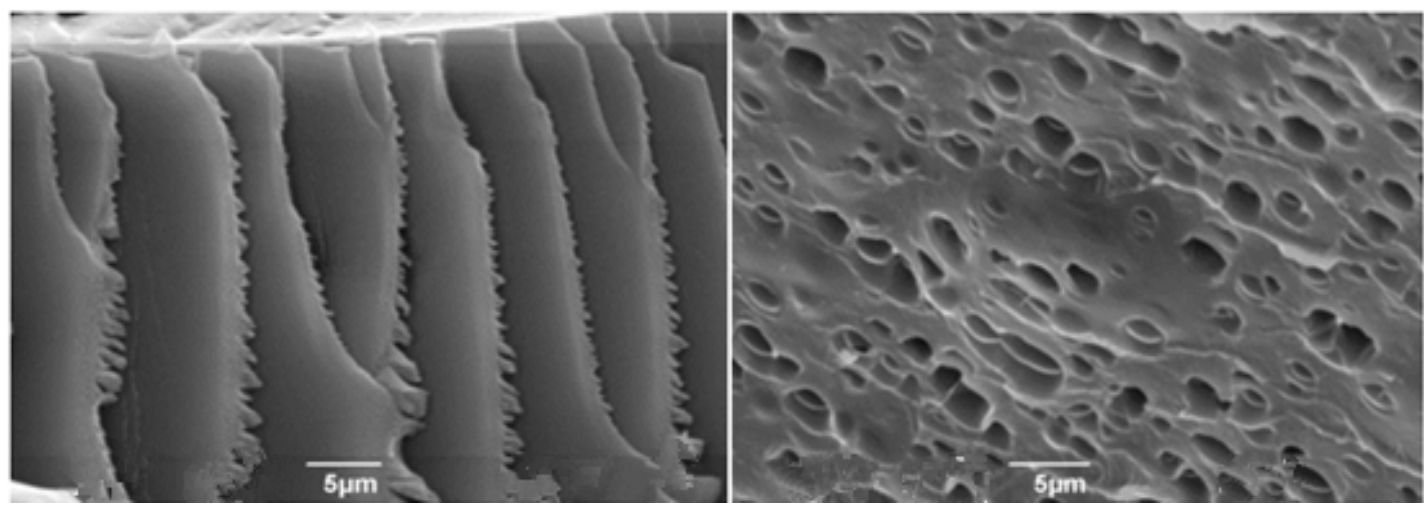

Figure 3. SEM images of PAM+PNipam hydrogel at a temperature higher than the critical temperature of $30^{\circ} \mathrm{C}$.

Combined with SEM images, it can be concluded that many small size porous structures will resist to PAM/PNipam long molecule collapsing in aqueous state, this small size (approximately 2-3um) is sufficient to trap some large suspended particulate matter in water. The thermal response hydrogels change state according to the change of temperature. When the temperature is higher than the critical temperature Tc, the thermal response gel changes phase and becomes a porous material, as shown in Figure 3. When the temperature is lower than this critical temperature, the thermal response layer returns to the hydrogel state, at which point the captured large-size suspended particulates, thereby achieving the purpose of filtering water residues.

Table 1. Various concentration of Nipam in hydrogel influenced in thermoresponsive performance.

\begin{tabular}{clcc}
\hline Ratio & Optimum TEMED & Transparent & Efficient \\
\hline $10 \%(\mathrm{w} / \mathrm{v})$ Nipam & $1 \mathrm{~mL}-2 \mu \mathrm{L}$ & $\mathrm{Y}$ & $\mathrm{Y}$ \\
$5 \% \mathrm{AM}$ & $1 \mathrm{~mL}-1 \mu \mathrm{L}$ & $\mathrm{Y}$ & $\mathrm{Y}$ \\
$5 \%$ Nipam+5\%AM & $1 \mathrm{~mL}-2.5 \mu \mathrm{L}$ & $\mathrm{Y}$ & $\mathrm{N}$ \\
$10 \%$ Nipam $+5 \% \mathrm{AM}$ & $1 \mathrm{~mL}-3.5 \mu \mathrm{L}$ & $\mathrm{Y}$ & $\mathrm{N}$ \\
$15 \%$ Nipam $+5 \% \mathrm{AM}$ & $1 \mathrm{~mL}-4.5 \mu \mathrm{L}$ & $\mathrm{Y}$ & $\mathrm{N}$ \\
$20 \%$ Nipam $+5 \% \mathrm{AM}$ & $1 \mathrm{~mL}-5 \mu \mathrm{L}$ & $\mathrm{Y}$ & $\mathrm{N}$ \\
$10 \%$ Nipam $+2.5 \% \mathrm{AM}$ & $1 \mathrm{~mL}-3 \mu \mathrm{L}$ & $\mathrm{Y}$ & $\mathrm{Y}$ \\
\hline
\end{tabular}

\subsection{Photocatalyst Thermal Response and Photochemical Response}

In some case, PAM/PNipam hydrogel is transparent and does not possess thermal response property. To implement the thermal response to PAM/PNipam hydrogel, we add a specific amount of EPE molecules to the precursor solution. The concentration of EPE molecules affects the degree of deepness of the blue color and its phase separation process. This is because the concentration of hydrogen ion is related to forming enough clusters to achieve absrobance UV block. By increasing the EPE concentration, hydrogel can turn entirely opaque to block shorter wavelength at uv region. Meanwhile, the concentration of tungstate and citrate acid has the similar effect on hydrogel. The initial state of which, is showed colorless observed by naked eyes and the cloudiness becomes more severe at higher concentration of EPE.

The UV-visible transmission spectrum of hydrogel made from $2.5 \%$ (w/v) AM monomer and $0.9 \%$ $(\mathrm{w} / \mathrm{v})$ EPE is shown in Figure 4. Comparing with DI water, the transmittance of hydrogel was comparable at $20^{\circ} \mathrm{C}$, hydrogel appeared almost transparent. As the temperature increases, the transmittance of hydrogel reduces gradually (Figure $4 \mathrm{a}$ ). When the temperature is above $30^{\circ} \mathrm{C}$, the transmittance approaches zero as shown in Figure 4a, the hydrogel appeared to be cloudy. This is 
because the thermal responsive hydrogel undergoes a gelation process. We analyzed the absorption spectrum of this catalytic material.

From the absorption spectrum, it can be seen that when the temperature is higher than $33^{\circ} \mathrm{C}$, the solution becomes milky white, and light cannot pass through at this time. Therefore, the absorption value has no practical significance, but it shows that the light passing rate is small. As the cooling process progresses, the solution gradually returns into transparent, and the solution is substantially transparent when cooled for 9 minutes.

When the finally formed photocatalyst was exposed at AM 1.5, the solution turned from colorless to blue due to the effect of ammonium tungstate (Figure $4 \mathrm{~b}$ ).

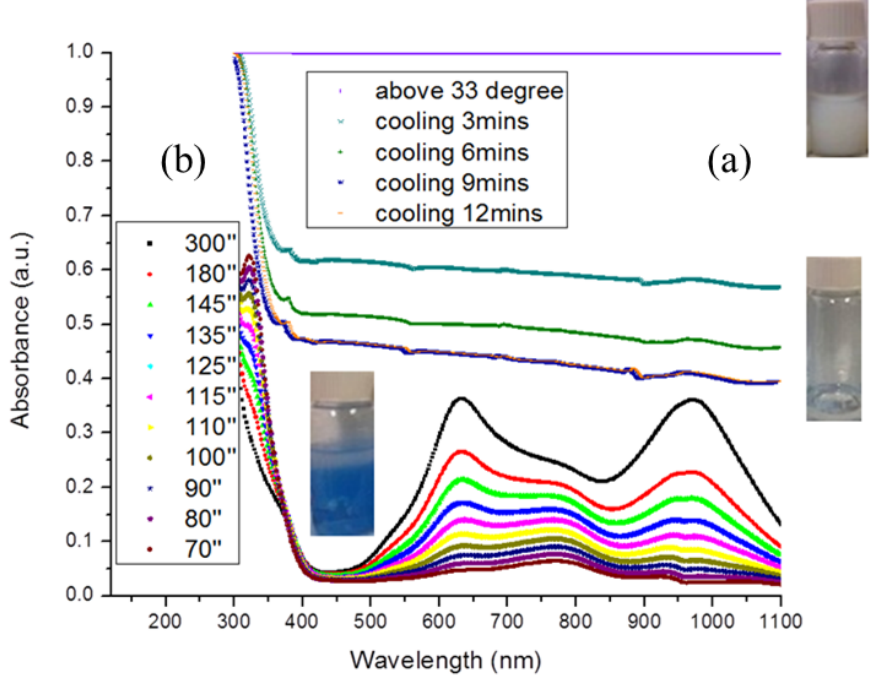

Figure 4. The UV-Vis transmission spectra of $5 \%(\mathrm{w} / \mathrm{v})$ polyacrylamide gel containing $0.9 \%(\mathrm{w} / \mathrm{v})$ EPE molecules at different temperatures.

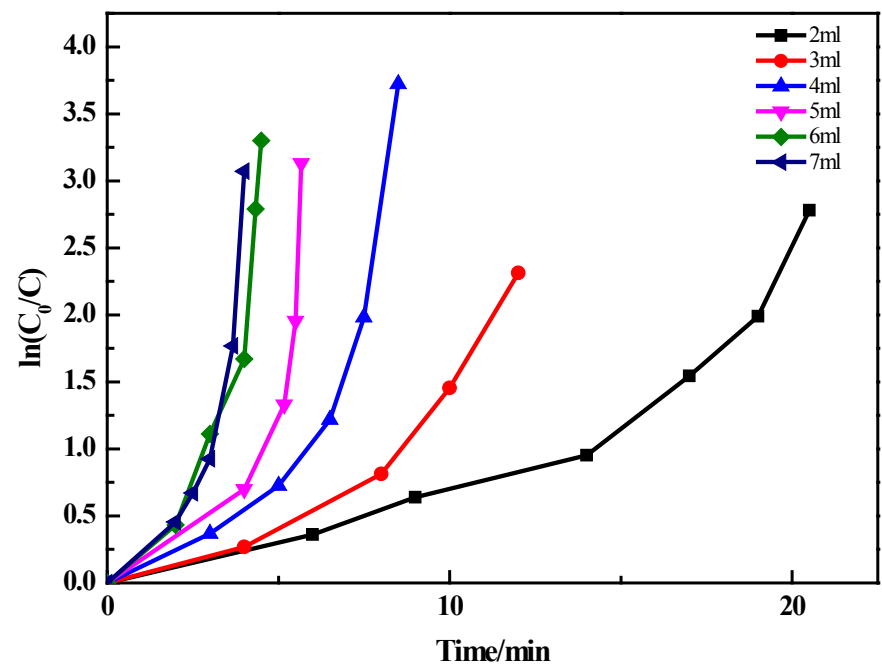

Figure 5. Photodecomposition of methyl orange dye in solution.

As the exposure time increases, the solution gradually turns blue, but there is no turbidity and no cross-linking process. It can be seen in the spectrum that the absorbance of the blue band gradually increases. The photooxidation capability of this photocatalyst was evaluated by measuring the decomposition of methyl orange (MO) dye in the MO solution (with concentration of $20 \mathrm{mg} / \mathrm{L}$ ), we 
adjust the addition amount of photocatalyst to obtain optimal catalytic efficiency. The experimental results are shown in Figure 5. As we can see, with the increasing of the amount of catalyst, the catalytic efficiency of methyl orange degradation accelerates too. Considering the cost, feasibility, the utilization rate of light, the separation of catalyst from water after the end of the reaction, and we can see that when the catalyst is added to $7 \mathrm{~mL}$, the degradation rate of methyl orange is not significantly improved, so we chose $6 \mathrm{~mL}$ as the value to evaluate the performance of this catalyst. The photocatalytic yield is about $0.084 \%$, indicating that the photocatalyst synthesized by this method has a good effect on degrading methyl orange. This may be due to the fact that the tungsten trioxide catalyst is well supported on the thermal responsive hydrogel, resulting in uniform dispersion of the photocatalyst, increase chance of contact with the methyl orange molecules, and improve catalytic efficiency, making the methyl orange degrade in a relatively short time. The internal degradation rate reached $96.3 \%$, and methyl orange was almost completely converted to other substances. It indicates that this nanocomposite thermoresponsive hydrogel can be used for wastewater treatment.

\section{Conclusion}

In this work, we prepared a new type of composite material with distinct thermal and light responsive characteristics as a functional material for wastewater purification. The substrate material is a thermal responsive hydrogel, and the photocatalysts with new photoresponsive properties aretungsten trioxide nanoparticles that are dispersed uniformly on a hydrogel. A network of porous fibers is formed by Nisopropylacrylamide (Nipam) and acrylamide (AM). Nipam/AM hydrogels undergo phase transformation when the temperature reaches the critical temperature. The pores formed when the hydrogel is cured are relatively uniform, this small size (approximately 2-3 um) is sufficient to trap some large suspended particulate matters in water, thereby achieving the purpose of filtering water residues.

By adding isopolytungstate nanoparticles as photocatalyst monomers, small-sized toxic organic materials can be degraded. The photocatalytic yield is about $0.084 \%$, indicating that the catalyst synthesized by this method has a good effect on degrading methyl orange and can be used for wastewater treatment. This new type of thermal and sunlight-sensitive hydrogel can be used as a functional material for the degradation of dyestuffs in wastewater and for the application of a large number of residues. It can be applied to environmental waste disposal and energy consumption.

Acknowledgments. The work was supported by the University of Science and Technology Beijing. The author thanks the teachers of Hong Kong University of Science and Technology and Beijing University of Technology for continuous support.

\section{References}

1. Shao, Penghui, et al. "Eco-friendly one-pot synthesis of ultradispersed $\mathrm{TiO}_{2}$ nanocrystals/graphene nanocomposites with high photocatalytic activity for dye degradation." Journal of Materials Chemistry A, vol. 3, no. 39, pp. 19913-19919, 2015.

2. Wang, J, et al. "Origin of photocatalytic activity of nitrogen-doped $\mathrm{TiO}_{2}$ nanobelts." Journal of the American Chemical Society, vol. 131, no. 34, pp. 12290-7, 2009.

3. Mioduska J, Janczarek M, Hupka J. "The Effect of Calcination Temperature on Structure and Photocatalytic Properties of $\mathrm{WO}_{3} / \mathrm{TiO}_{2}$ Nanocomposites." Journal of Nanomaterials, vol. 2016, no. 9, pp. 1-8, 2016.

4. Peng, Wen Chao, and X. Y. Li. "Synthesis of $\mathrm{MoS}_{2} / g-\mathrm{C}_{3} \mathrm{~N}_{4}$, as a solar light-responsive photocatalyst for organic degradation." Catalysis Communications, vol. 49, no. 5, pp. 63-67, 2014.

5. Gan, Lu, et al. "Visible light induced methylene blue dye degradation photo-catalyzed by WO3 /graphene nanocomposites and the mechanism." Ceramics International, vol. 42, no. 14, pp. 15235-15241, 2016.

6. Sayama, Kazuhiro, et al. "Photocatalytic and Antibacterial Activities over $\mathrm{WO}_{3}$ on Glass Filters." Chemistry Letters, vol. 39, no. 8, pp. 884-885, 2010.

7. Zhang, Gehong, et al. "Organic Additives-Free Hydrothermal Synthesis and Visible-Light-Driven Photodegradation of Tetracycline of $\mathrm{WO}_{3}$ Nanosheets." Industrial \& Engineering Chemistry Research, Vol. 53, no. 13 , pp. 5443-5450, 2014. 
8. Jia, Qingxin, K. Iwashina, and A. Kudo. "Facile fabrication of an efficient $\mathrm{BiVO}_{4}$ thin film electrode for water splitting under visible light irradiation." Proceedings of the National Academy of Science, vol. 109, no. 29, pp. 11564-11569, 2012.

9. Nosaka, Yoshio, and A. Y. Nosaka. "Generation and Detection of Reactive Oxygen Species in Photocatalysis." Chemical Reviews, vol. 117, no. 17, 2017.

10. Wang, Peng, et al. "Ag/ $\mathrm{AgBr} / \mathrm{WO}_{3} \cdot \mathrm{H}_{2} \mathrm{O}$ : Visible-Light Photocatalyst for Bacteria Destruction." Inorganic Chemistry, vol. 48, no. 22, pp. 10697-10702, 2009.

11. Vargasconsuelos, C. Ingram, et al. "Correlation between Particle Size and Raman Vibrations in $\mathrm{WO}_{3}$ Powders." Journal of Physical Chemistry C, vol. 118, no. 18, pp. 9531-9537, 2014.

12. Ma, Yi, et al. "Titanium Dioxide-Based Nanomaterials for Photocatalytic Fuel Generations." Chemical Reviews, vol. 114, no. 19, pp. 9987-10043, 2014.

13. Xiang, Q., et al. "Au Nanoparticle Modified $\mathrm{WO}_{3}$ Nanorods with Their Enhanced Properties for Photocatalysis and Gas Sensing." Journal of Physical Chemistry C, vol.114, no. 5, pp. 2049-2055, 2010.

14. Jungwon, Kim, L. Chulwee, and C. Wonyong. "Platinized $\mathrm{WO}_{3}$ as an environmental photocatalyst that generates $\mathrm{OH}$ radicals under visible light." Environmental Science $\&$ Technology, vol. 44, no. 17, pp. 6849, 2010.

15. Yin, H., et al. "Ag/BiOBr Film in a Rotating-Disk Reactor Containing Long-Afterglow Phosphor for Round-theClock Photocatalysis." Acs Appl Mater Interfaces, vol. 7, no. 36, pp. 20076-20082, 2015.

16. Bai, Yang, et al. "Enhanced photocatalytic performance of direct Z-scheme BiOCl-g- $\mathrm{C}_{3} \mathrm{~N}_{4}$ photocatalysts." Rsc Advances, vol. 4, no. 37, pp. 19456-19461, 2014.

17. Rokhsat, Eliza, and O. Akhavan. "Improving the photocatalytic activity of graphene oxide/ZnO nanorod films by UV irradiation." Applied Surface Science, vol. 37no. 1, pp. 590-595, 2016.

18. Nishikawa M, Mitani Y, Nosaka Y. "Photocatalytic Reaction Mechanism of Fe(III)-Grafted $\mathrm{TiO}_{2}$ Studied by Means of ESR Spectroscopy and Chemiluminescence Photometry." Journal of Physical Chemistry C, vol. 116, no. 28, pp. 14900-14907, 2012.

19. Akhavan, O. "Lasting antibacterial activities of $\mathrm{Ag}-\mathrm{TiO}_{2} / \mathrm{Ag} / \mathrm{a}-\mathrm{TiO}_{2}$ nanocomposite thin film photocatalysts under solar light irradiation." Journal of Colloid \& Interface Science, vol. 336, no. 1, pp. 117-124, 2009.

20. Etacheri, Vinodkumar, et al. "A Highly Efficient $\mathrm{TiO}_{2}-\mathrm{xCx}$ Nano-heterojunction Photocatalyst for Visible Light Induced Antibacterial Applications." Acs Applied Materials \& Interfaces, vol. 5, no. 5, pp. 1663-1672, 2013.

21. Xi, G., et al. " $\mathrm{Fe}_{3} \mathrm{O}_{4} / \mathrm{WO}_{3}$ hierarchical core-shell structure: high-performance and recyclable visible-light photocatalysis." Chemistry, vol. 17, no. 18, pp. 5145-5154, 2011.

22. Yoo, M. K., et al. "Effect of polyelectrolyte on the lower critical solution temperature of poly (N-isopropyl acrylamide) in the poly (NIPAAm-co-acrylic acid) hydrogel." Polymer, vol. 41, no.15, pp. 5713-5719, 2000.

23. Tripathi, A, and A. Kumar. "Multi-featured macroporous agarose-alginate cryogel: synthesis and characterization for bioengineering applications." Macromolecular Bioscience, vol. 11, no.1, pp. 22-35, 2011.

24. Orive, G, et al. "Survival of different cell lines in alginate-agarose microcapsules." European Journal of Pharmaceutical Sciences Official Journal of the European Federation for Pharmaceutical Sciences, vol. 18, no.1, pp. 23-30, 2003.

25. Feng, Yibo, et al. "Thermally Responsive Composite Hydrogel via Self-Assembly for Smart Window Applications." Journal of Nanomaterials, vol. 11, no. 1, pp. 1-9, 2016. 